\title{
MPC Using Modulated Optimal Voltage Vector for Voltage Source Inverter with LC Output Filter
}

\author{
Shafiq Odhano, Pericle Zanchetta, Mi Tang \\ Department of Electrical and Electronic Engineering \\ The University of Nottingham \\ Nottingham, United Kingdom
}

\author{
Cesar A. Silva \\ Departamento de Electrónica \\ Universidad Técnica Federico Santa María \\ Valparaíso, Chile
}

\begin{abstract}
Voltage source inverters with LC output filters are widely used for high-quality output of ac power supplies. They are also a potential solution for embedded electrical networks in more-electrical aircraft of the future. In this paper, a recently developed model predictive control technique that selects modulated optimal voltage vector is applied for high dynamic output voltage control of these systems especially when the required output frequency is well above the standard $50 / 60 \mathrm{~Hz}$ as required by aerospace applications. The studied predictive control optimizes the switch duty cycles when in linear regulation range by solving analytical equations. The over-modulation region is covered by optimized linear combination of the adjacent hexagon vertices unlike how it happens in classical space vector modulation. A load current observer is also designed in this paper which is made independent of output frequency for improving the disturbance rejection capability of the voltage control loop when the load current sensors are not present. Simulation and experimental results that validate the control and observer performance are presented under different steady state and dynamic operating conditions.
\end{abstract}

Index Terms-LC filters, load current observers, modulated model predictive control, non-linear loads, optimal vectors, voltage source inverters

\section{INTRODUCTION}

Voltage source inverters (VSI) with output inductivecapacitive (LC) filters are most commonly employed systems for applications such as interfacing renewable energy sources with ac microgrids [1] and uninterruptible power supplies [2]. Additionally, in drive applications the LC filter can be used to improve waveform quality [3], [4]. The requirement of a ripple-free voltage at load terminals is the raison d'être of an LC filter at the output of a VSI [3]. For an ideal ac power supply, it is imperative that the quality of output be independent of the type of load as long as the power ratings are not exceeded. With non-linear loads, it becomes the merit of the controller to keep the distortions in the output voltage to a minimum. A fast dynamic control with rapid disturbance rejection improves the overall quality of any ac power supply while maintaining a clean output voltage. However, the inclusion of an LC filter renders the control design procedure a bit involved and parameter dependence and axes decoupling become major issues.

The output voltage control of a VSI with LC filter is still a subject of research especially when it comes to control dynamics [5]-[13]. Model predictive control (MPC) has been shown to be a suitable alternative to linear controllers due to the former's superior dynamics over the latter [14]. In power electronics, its use has attracted much attention in the recent past [10], [15]-[18]. Both finite control set (FCS) [5] MPC as well as its modulated alternative [19], [20] have been the focus of research. A growing trend towards modulated optimum voltage vector based MPC is the motivation behind this work.

Voltage control of a VSI equipped with an output LC filter is studied in depth in the literature. For instance, [9] proposes a robust control scheme for a VSI interfaced with the grid through an LC filter. The authors have dealt with the problem of changing grid impedance and have proposed an $\mathcal{H}_{\infty}$ controller for robustness. The implications of state feedback coupling on voltage control are analysed in [8] and some ideal and non-ideal decoupling strategies are introduced. A comprehensive analysis of multiloop control strategies for VSI and current source inverter (CSI) equipped with output LC, CL, or LCL filters is studied in [13]. In [12], a generalized closed loop control of VSI with LC and LCL output filters is proposed using the concept of internal and external virtual impedances. An FCS-MPC based approach is used to achieve output voltage control in [5]. A cost-function is defined as the difference between required and predicted output voltage and the inverter switching state that minimizes this difference is applied during the next switching interval. To mitigate the impact of load current, a load current observer is also designed. However, the implementation takes place in $\alpha \beta$ stationary reference frame that makes the observer response frequency dependent. The work presented in [21], redesigns the load current observer for sinusoidal load currents while employing the same control strategy as presented in [5] effectively reducing the impact of output frequency on observer response. MPC for the output voltage control of a four-leg VSI with LC output filter is presented in [10] in which the cost function also includes a term to reduce the switching frequency of the neutral (fourth) leg.

The implementation of MPC through a modulator has recently been introduced due to its merits of constant switching frequency; it has also been shown to eliminate the limitations of FCS-MPC when working with low reactance machines [20]. The modulated optimal vector based control of a VSI with LC filter is presented in [6] in which the authors have computed explicit optimum solution when the dc-link voltage constraint 
is not violated. In case the optimum lies outside the bounds of a VSI's available dc-link voltage, it is scaled down to lie along the constraint border. Another optimal voltage vector based MPC is proposed for a permanent magnet synchronous motor's speed control in [22] where the duty cycles for space vector modulation (SVM) are explicitly calculated by solving an analytical equation. In the linear regulation range the calculated duty cycles are directly applied to the inverter switches, as in [6], whereas in over-modulation the duties are reduced through a linear combination to keep the optimality condition. A closely similar strategy is employed in [23] that optimizes the command voltage vector both in linear regulation and in overmodulation range, however, differently from [22] the approach presented in [23] identifies the optimum and second-optimum vectors from the cost-function evaluation.

In this paper, the modulated MPC strategy of [22] and [23] is modified for its application to the output voltage control of a VSI with LC filter. The implementation is carried out in the $d q$ rotating reference frame as opposed to $\alpha \beta$ frame as done in [22] and [5]. A load current observer is designed, again in the $d q$ frame, to cancel load disturbance effect on the control performance when load current is not measured. It is demonstrated that the observer dynamics are independent of output frequency which renders the method suitable for applications such as modern programmable ac power supplies [24] or for open-loop control of an induction machine [3]. The method is verified in simulation and through experimental analysis with different control objectives and performance merits.

The paper is organized as follows. In section II the mathematical modelling of a VSI with LC filter is presented along with the load current observer, section III introduces the control strategy used, section IV shows control performance results and, finally, section $\mathrm{V}$ concludes the paper.

\section{VSI WITH LC FILTER: MODELLING}

Fig. 1 shows a VSI with output LC filter and a generic threephase load. In this figure, $L$ is filter inductance per phase, $R_{L}$ is its series resistance and $C$ is filter capacitance. The vector $\bar{i}_{L}$ denotes current through the inductors and vector $\bar{v}_{c}$ is the voltage across filter capacitances; these two are the state variables of the system and are measured through current and voltage sensors, respectively. The figure also shows the load current vector $\bar{i}_{o}$, which is not a state variable and is not a measured quantity. The instantaneous voltage vector applied by the inverter is given by the state of each switch S1-S6 and is denoted by $\bar{u}_{i}$ in the following state equations written in $d q$ rotating reference frame where $\omega$ is the radian speed of this frame.

$$
\begin{gathered}
\frac{d \bar{i}_{L}}{d t}=-\frac{R_{L}}{L} \bar{i}_{L}-j \omega \bar{i}_{L}+\frac{1}{L}\left(\bar{u}_{i}-\bar{v}_{c}\right) \\
\frac{d \bar{v}_{c}}{d t}=-j \omega \bar{v}_{c}+\frac{1}{C}\left(\bar{i}_{L}-\bar{i}_{o}\right)
\end{gathered}
$$

Eq. (1) and (2) give the state equations of the VSI with LC filter where the load current $\bar{i}_{o}$ is an unknown input.

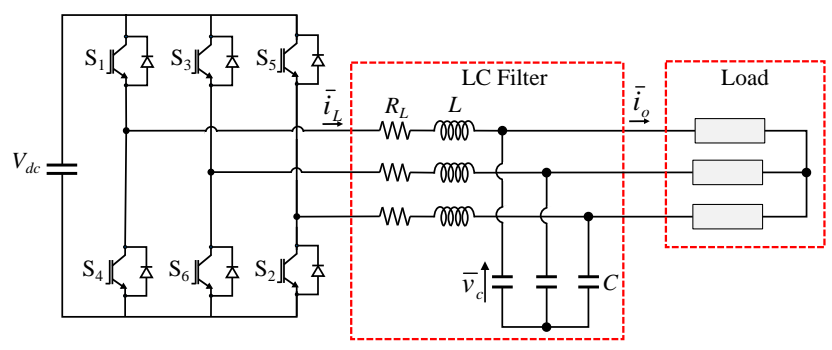

Fig. 1. VSI with LC output filter supplying a generic three-phase load.

Nevertheless, it can be obtained through a properly designed load current observer. It is assumed that the load current has slower dynamics than $\bar{i}_{L}$ and $\bar{v}_{c}$ such that $\dot{\bar{i}}_{o}=0$. With this assumption, the following state and output equations can be written where $\bar{i}_{L}, \bar{v}_{c}$, and $\bar{i}_{o}$ are vectors in $d q$ reference frame.

$$
\begin{gathered}
\frac{d}{d t}\left[\begin{array}{l}
\bar{i}_{L} \\
\bar{v}_{c} \\
\bar{i}_{o}
\end{array}\right]=\underbrace{\left[\begin{array}{ccc}
-\frac{R_{L}}{L}-j \omega & \frac{1}{L} & 0 \\
\frac{1}{C} & -j \omega & -\frac{1}{C} \\
0 & 0 & 0
\end{array}\right]}_{A} \underbrace{\left[\begin{array}{c}
\bar{i}_{L} \\
\bar{v}_{C} \\
\bar{i}_{o}
\end{array}\right]}_{\underline{x}}+\underbrace{\left[\begin{array}{c}
1 \\
L \\
0 \\
0
\end{array}\right]}_{B} \bar{u}_{i} \\
y=\underbrace{\left[\begin{array}{lll}
1 & 0 & 0 \\
0 & 1 & 0
\end{array}\right]}_{C} \underline{x}
\end{gathered}
$$

Eq. (3) combines the two state equations (1) and (2) and includes the third equation for load current with the condition $\dot{\bar{i}}_{o}=0$. Additionally, the output of the system is defined in (4) by $\underline{y}=C \underline{x}$.

A state observer can be defined from (3) and (4) as:

$$
\underline{\dot{\hat{x}}}=A \underline{\hat{x}}+B \underline{u}+K(\underline{y}-\hat{\hat{y}})
$$

with $K$ as observer gain matrix. The error dynamics of this observer can be derived as:

$$
\underline{\dot{e}}=(A-K C) \underline{e}
$$

The eigenvalues of $(A-K C)$ give the poles of the observer. The observer gain matrix $K$ can be obtained through eigenvalue assignment method that places the observer poles at desired locations on the complex plane. It can be observed from (3) that with a fixed gain $K$, the poles of the observer move with the rotating reference frame's angular speed $\omega$. However, if the arbitrary $\omega$ is put equal to zero, the problem is conducted back to the stationary $\alpha \beta$ frame. Furthermore, if $R_{L}$ is also neglected, the observer becomes identical to the one proposed in [5]. In order to compute the observer gain matrix $K$, it is assumed that $\omega=0$ and $R_{L}=0$ but in the structure of the observer the terms containing $\omega$ are retained to decouple the $d$ and $q$ axes. This strategy allows to design the observer through a traditional pole placement method while keeping it independent of the rotating reference frame's angular frequency $\omega$. If the observer poles were to be placed at $-\omega_{0}\left[\mu_{1},\left(\mu_{2}+j \mu_{2}\right),\left(\mu_{2}-j \mu_{2}\right)\right]$ with $\left(\mu_{1}, \mu_{2}\right)>0$ then the observer gains, through eigenvalue assignment, would be: 


$$
K=\left[\begin{array}{cc}
\mu_{1} \omega_{0} & -\frac{1}{L} \\
\frac{1}{C} & 2 \mu_{2} \omega_{0} \\
0 & -2\left(\mu_{2} \omega_{0}\right)^{2} C
\end{array}\right]
$$

It can be noticed from (7) that the observer gains can be computed a priori based only on the LC filter parameters and independently of $\omega$. A discussion on the evolution of observer poles as a function of $\omega$ is given in the latter sections.

\section{Model Predictive Control with Modulated OPTIMUM VECTORS}

To be able to apply any prediction based control scheme in a digital environment, the first step is to obtain the discrete equations of the controlled system. The equations (1) and (2) are discretized using Euler's approximation as (8) and (9), respectively, where $k$ and $k+1$ signify values at consecutive sampling instants separated by sample time $T_{s}$.

$$
\begin{gathered}
\bar{i}_{L}(k+1)=\left(1-\frac{R_{L}}{L} T_{s}-j \omega T_{s}\right) \bar{i}_{L}(k)+\frac{T_{s}}{L}\left(\bar{u}_{i}(k)-\bar{v}_{c}(k)\right) \\
\bar{v}_{c}(k+1)=\left(1-j \omega T_{s}\right) \bar{v}_{c}(k)+\frac{T_{s}}{C}\left(\bar{i}_{L}(k)-\bar{i}_{o}(k)\right)
\end{gathered}
$$

To account for the digital implementation delay, (8) and (9) are evaluated twice [25]. First, $\bar{i}_{L}(k+1)$ is predicted from the previous command voltage $\bar{u}_{i}(k)$ and measured $\bar{v}_{c}(k)$ and $\bar{v}_{c}(k+1)$ is predicted from measured $\bar{i}_{L}(k)$ and observed $\bar{i}_{o}(k)$. Then, in the second instant, (8) is evaluated for all possible inverter states for which the voltage $\bar{u}_{i}$ in $\alpha \beta$ frame is given by (10) and in rotating $d q$ reference frame by (11).

$$
\bar{u}_{i \alpha \beta}=\frac{2}{3} V_{d c}\left[\begin{array}{lll}
1 & e^{j \frac{2 \pi}{3}} & e^{j \frac{-2 \pi}{3}}
\end{array}\right]\left[\begin{array}{l}
S_{a} \\
S_{b} \\
S_{c}
\end{array}\right]
$$

where $V_{d c}$ is the available dc-link voltage, $S$ denotes switching function with subscripts ' $a$ ', ' $b$ ', and ' $c$ ' representing phases. The top switch of the inverter leg is closed for $S=1$ i.e. $S_{a}=1$ corresponds to $S_{1}=1$ and $S_{4}=0$ in Fig. 1 .

$$
\bar{u}_{i}=\bar{u}_{i \alpha \beta} e^{-j \theta}
$$

where $\theta$ is the angle of the rotating reference frame given by (12).

$$
\theta=\int \omega d t
$$

At this point, using the predicted inductor current $\bar{i}_{L}(k+2)$ and observed load current $\hat{\bar{i}}_{o}$, the capacitor (or load) voltage is predicted for the next sampling instant (for all seven inverter states). The predicted $\bar{v}_{c}$ is compared with the reference load voltage $\left(\bar{v}_{c}^{*}\right)$ and an error vector is computed as (13). Notice that the error vector has seven elements (each of them a complex number).

$$
\bar{E}_{i}=\bar{v}_{c}^{*}-\bar{v}_{c}(k+2)
$$

where the subscript ' $i$ ' denotes the inverter state $i \in[0,1, \ldots, 6]$.
The two error vectors that have minimum magnitude are identified as $\bar{E}_{1}$ and $\bar{E}_{2}$ using either the geometrical analysis of [22] or simply by minimum magnitude and adjacency criteria of [23]. It should be noted that the real part of the error vector corresponds to the $d$-axis component and the imaginary part to its $q$-axis component. The inverter switching indices corresponding to these two error vectors are selected as candidate voltage vectors to be applied at the next sampling instant. However, the duty cycles $d_{1}$ and $d_{2}$ with which to apply these two vectors is computed by solving the system of linear equations (14) where $\bar{E}_{0}$ is the error vector when null state of the inverter is applied (i.e. all switches closed on positive dc-link or on its negative terminal).

$$
\left[\begin{array}{ll}
E_{1 d}-E_{0 d} & E_{2 d}-E_{0 d} \\
E_{1 q}-E_{0 q} & E_{2 q}-E_{0 q}
\end{array}\right]\left[\begin{array}{l}
d_{1} \\
d_{2}
\end{array}\right]=\left[\begin{array}{l}
-E_{0 d} \\
-E_{0 q}
\end{array}\right]
$$

When in linear regulation range, the values of $d_{1}$ and $d_{2}$ produced by (14) satisfy $\left(d_{1}+d_{2}\right)<1$ and therefore the duty cycle for the zero state is obtained as $d_{0}=1-d_{1}-d_{2}$. If it is not possible to reach the reference voltage $\bar{v}_{c}^{*}$ in one sampling instant with the available dc-link voltage, (14) gives $\left(d_{1}+d_{2}\right)>1$ in which case the duty cycles are linearly scaled down as given by (15). This type of saturation is implemented in [22] and is a sub-optimal solution, although with a sufficiently fast response that converges to the desired solution. It must be noted that (15) preserves the fast dynamics of FCS-MPC for very large transient errors as demonstrated in [22]. In [23], this saturation is implemented based on an optimization (and a subsequent approximation) leading to a slightly different solution.

$$
d_{1}=\frac{d_{1}}{d_{1}+d_{2}} \quad d_{2}=1-d_{1} \quad d_{0}=0
$$

\section{EXPERIMENTAL AND SimUlation RESUlts}

The presented control strategy is implemented both in simulation and experiments for validation. Fig. 2 shows the experimental setup used for tests. The LC filter parameters are: $L=3.0 \mathrm{mH}, R_{L}=0.2 \Omega, C=40 \mu \mathrm{F}$. A balanced threephase resistive load with $R_{\text {load }}=10 \Omega$ is used. The control performance is evaluated for voltage step change and, unlike the results presented in the literature, the control response for a frequency step change is also analysed experimentally for completeness. The load current observer gains in (7) are set using $\omega_{0}=2 \pi \cdot 200, \mu_{1}=1$ and $\mu_{2}=1$. The switching frequency is $10 \mathrm{kHz}$, however in simulation average model of the inverter is implemented.

Before presenting results for validation of the proposed control and observer, it is necessary to check the observer stability for different output frequencies. As noted earlier, the observer gains are tuned based on the assumption $\omega=0$, however in the observer structure $\omega$ appears as a decoupling term (3). Once the observer gains are set according to (7), the evolution of observer poles for variable $\omega$ is analysed to determine stability. Even though the problem is that of complex-coefficient transfer functions and must be studied 


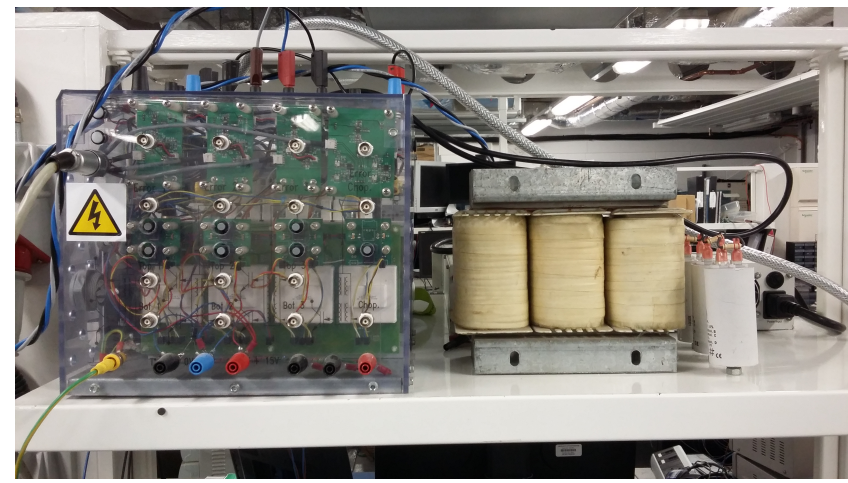

Fig. 2. Setup for experimental tests

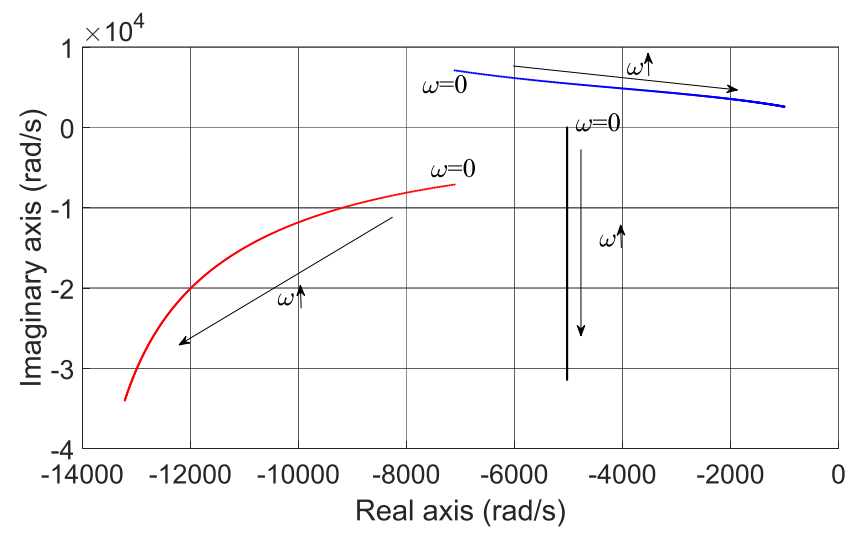

Fig. 3. Observer poles' location for $0<\omega<2 \pi \cdot 5000$

in depth [26], here it is sufficient to evaluate observer pole locations for a practical range of $\omega$. For instance, with a switching frequency of $10 \mathrm{kHz}$, the maximum output frequency of $3.2 \mathrm{kHz}$ can be obtained. Fig. 3 gives the locus of observer poles for $\omega$ sweep from 0 to $5 \mathrm{kHz}$. The poles remain on the left-half-plane of the complex plane that verifies stability (in a very rudimentary way).

Fig. 4 shows the controller response for a step change in voltage magnitude from zero to maximum possible that can be achieved with a dc-link of 200V. The lower plot in Fig. 4 shows the switch duty cycles of the top switches of Fig. 1. At the instant that step is applied, the duties saturate (indicating operation in over-modulation region) because of large error which cannot be eliminated in one switching instant. Fig. 5 top plot gives the $d$ - and $q$-axis reference and measured voltages, a slow build-up of voltage is because of the inverter current limit being reached when the voltage across filter capacitance is controlled. Note that the required output voltage is considered to be along $d$-axis (i.e. $v_{d}^{*}=V_{a m p}^{*}$ and $v_{q}^{*}=0$ ). As can be seen, the $q$-axis voltage is disturbed during the large transient of $d$-axis voltage step but is effectively brought to zero in steady state. The lower plot of Fig. 5 shows the duty cycles $d_{1}, d_{2}$ and $d_{0}$ given by (14) (in linear range) and by (15) (in over-modulation). At the moment a voltage step is applied, the duty cycles remain saturated for the initial instants and then
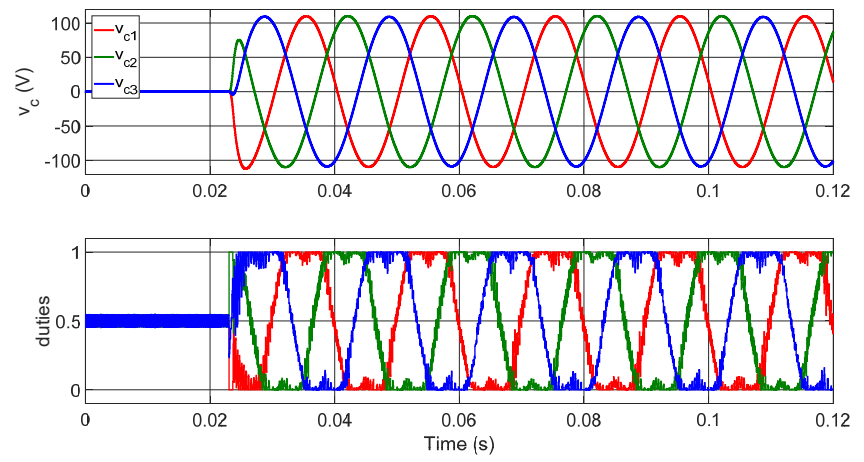

Fig. 4. Dynamic response for reference voltage magnitude step change - top: phase voltages across filter capacitances, bottom: phase duty cycles.
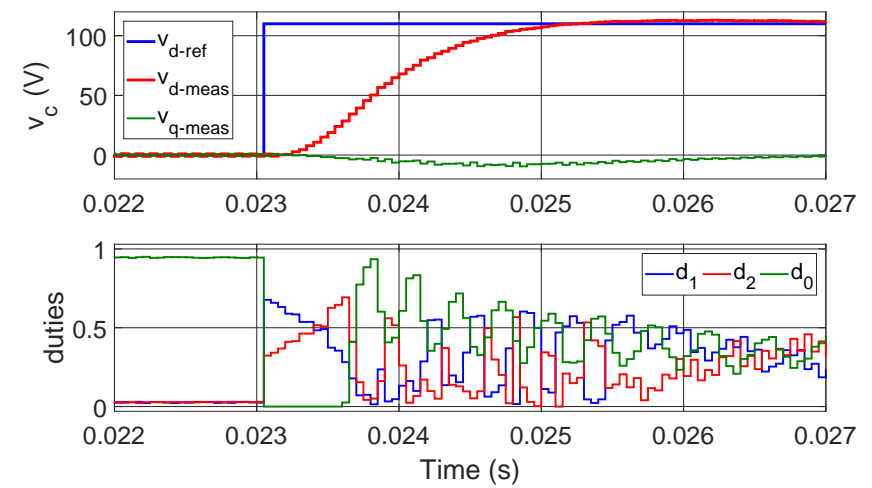

Fig. 5. $d q$-frame voltages and duties during voltage reference step - top: reference and measured $d$ - and $q$-axis load voltage, bottom: duty cycles for the two active and a zero vector given by (14) and (15).

the linear modulation starts. It must be noted that the duty cycles $d_{1}$ and $d_{2}$ are saturated in the sense that their sum is 1 but are linearly scaled as per (15). In the saturation region, the fast dynamic performance of FCS-MPC is preserved.

Fig. 6 presents the voltage control performance in the absence of load current measurement or observer. Since the modulated MPC implemented here calculates the exact solution for switch duty cycles through (14) with no integrative correction element, this results in the steady-state error seen in Fig. 6. The disturbance (load current) is not compensated and the exact solution is prone to steady-state error. In Fig. 7, the same voltage step condition of Fig. 6 is applied but this time with the load current observer included. As expected the steady-state error disappears because now the additive disturbance has been compensated.

Fig. 8 shows the results when a frequency step is applied at $t=0.132 \mathrm{~s}$. The reference frequency is changed from $50 \mathrm{~Hz}$ to $250 \mathrm{~Hz}$. However, the reference voltage magnitude is reduced for this test as the dc-link voltage is not sufficient to supply the load current at this frequency (due to higher voltage drop in filter inductance). The upper plot shows the measured load voltage and the lower plot gives the measured load current. In Fig. 9 the dynamics of the load current observer are tested when a step change in reference voltage of Fig. 4 is applied. The plot compares the observed phase current with 

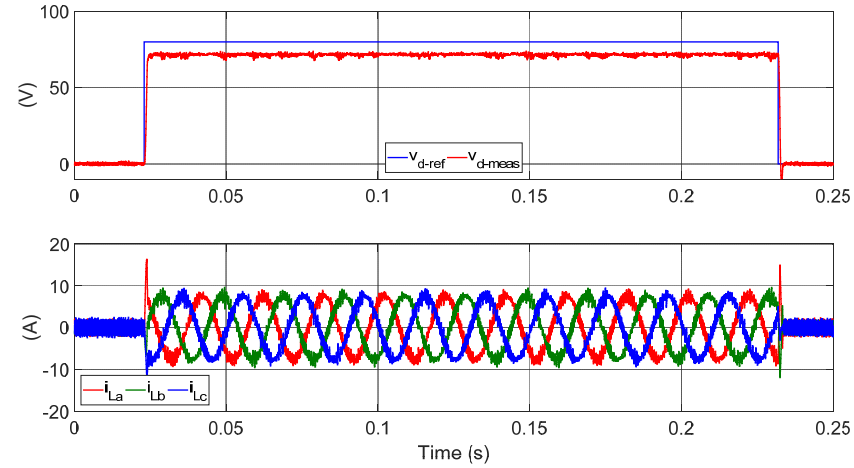

Fig. 6. Output voltage control without load current measurement or observer showing steady state error - top: reference and measured $d$-axis voltage, bottom: filter inductor phase currents.
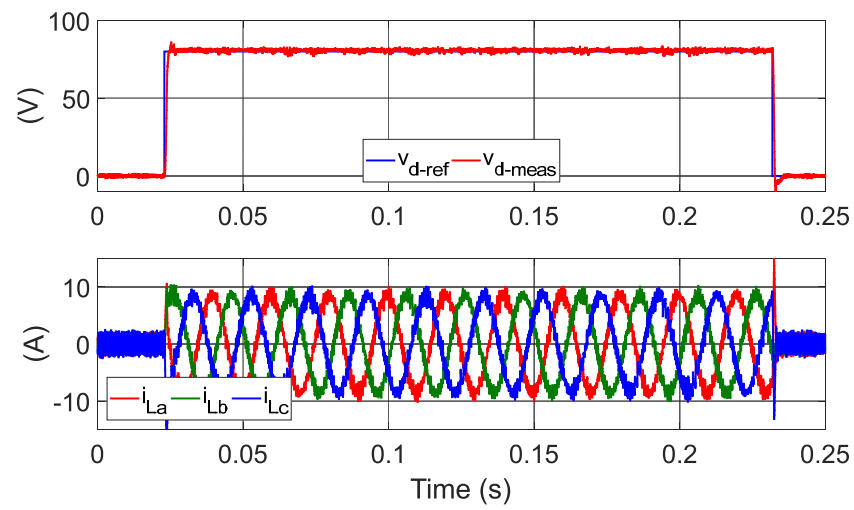

Fig. 7. Output voltage control with load current observer - top: reference and measured $d$-axis voltage, bottom: filter inductor phase currents.
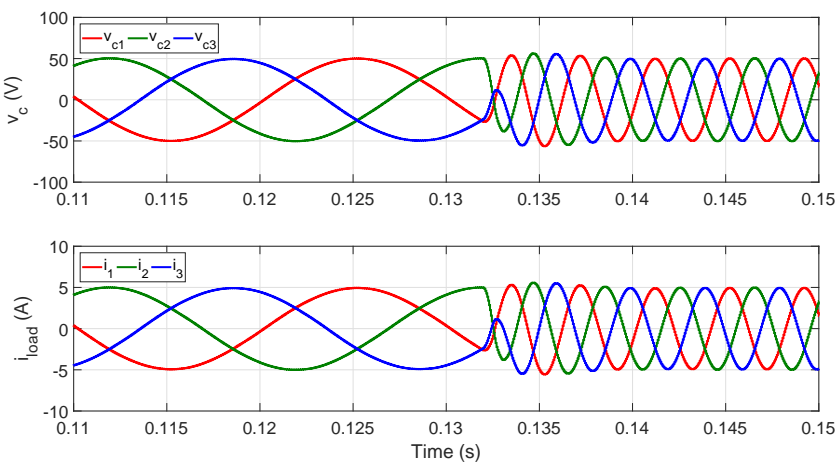

Fig. 8. Controller and observer dynamics with frequency step response - top: measured phase voltages at the load, bottom: observed load current.

the measured one. The observer dynamics under a frequency step change (of Fig. 8) are presented in Fig. 10 where again the observed current is compared with the measured phase current. The advantages of implementing the load current observer in rotating $d q$ reference frame are evident in this figure.

The operation under non-linear load condition is evaluated in simulation with a diode bridge rectifier shown in Fig. 11. The dc-side capacitance $C_{d c}$ is $1880 \mu \mathrm{F}$ and the load is a resistance of $100 \Omega$. Fig. 12 shows the control response for

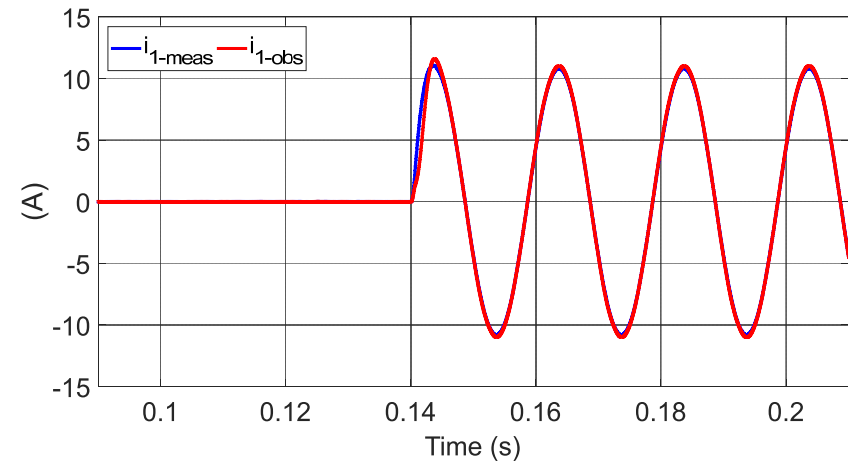

Fig. 9. Observer dynamics for load voltage magnitude step change comparison between measured and observed current of one load phase.

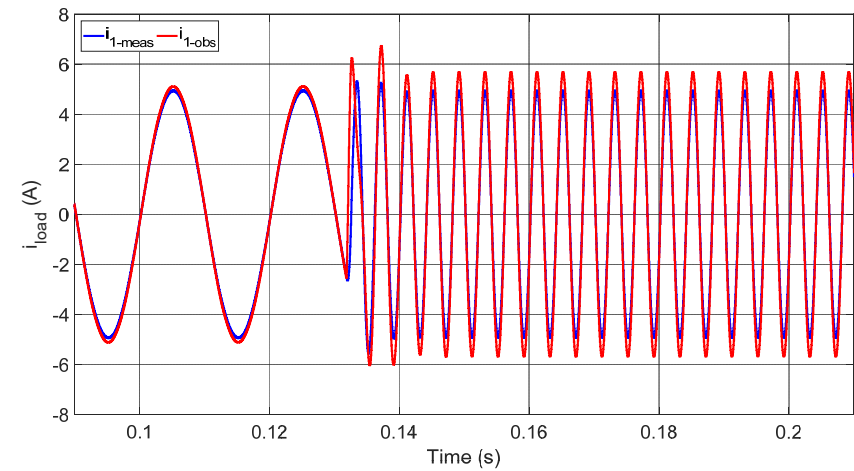

Fig. 10. Observer dynamics for output frequency step - comparison between measured and observed current of one load phase.

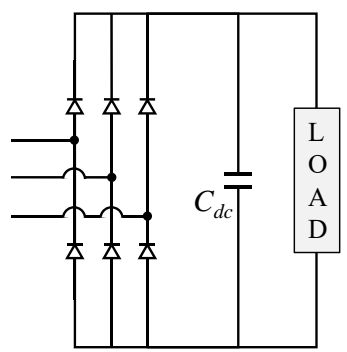

Fig. 11. Non linear load

a voltage step with the non-linear load of Fig. 11. It must be noted that to verify the control dynamics, the dc-link capacitance is pre-charged to avoid inrush currents following a reference voltage step. Although an output frequency change is not relevant with the load of Fig. 11, it is tested for the sake of completeness. Fig. 13 shows the results for a frequency step change from $50 \mathrm{~Hz}$ to $250 \mathrm{~Hz}$. To verify the control performance in a more challenging environment, a test is performed in which the load of Fig. 11 and a three-phase resistive load are connected in parallel. Fig. 14 shows the control performance for a load voltage step and Fig. 15 gives the results for frequency step.

Next, the dynamics of the observer designed in this paper are compared with that proposed in the literature [5]. The 

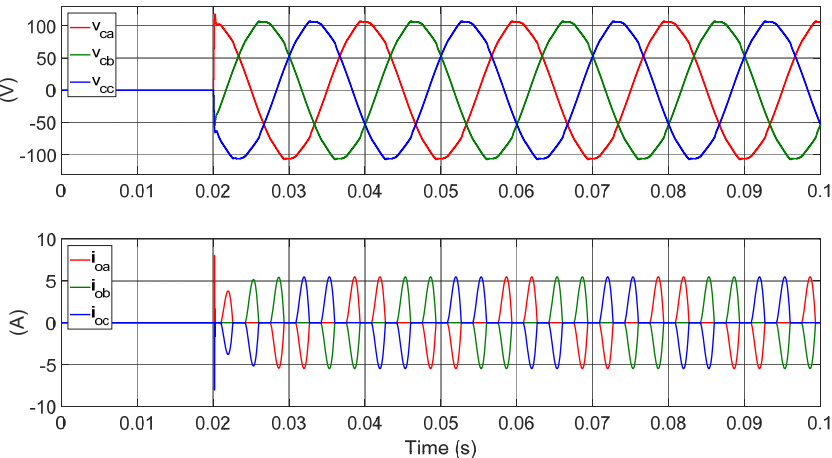

Fig. 12. Voltage step response with non-linear load - top: output phase voltages, bottom: load phase currents.
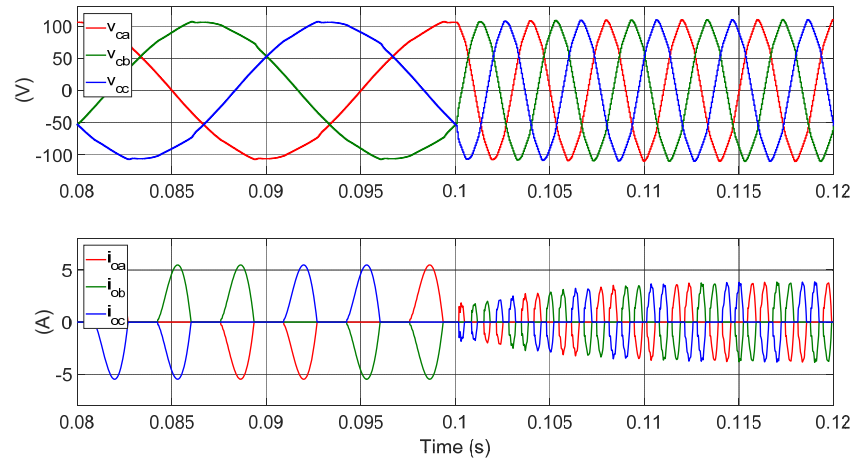

Fig. 13. Frequency step response with non-linear load - top: output phase voltages, bottom: load phase currents.
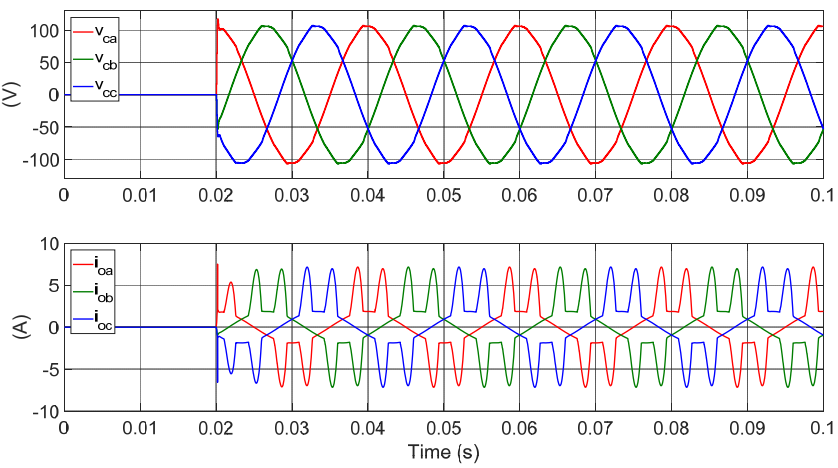

Fig. 14. Voltage step response with a combination of linear and non-linear loads - top: output phase voltages, bottom: load phase currents.

top plot in Fig. 16 demonstrates that the observer designed in this paper shows practically no dependence on the output frequency thanks to the decoupling terms containing $\omega$ in (3). The bottom plot of Fig. 16 highlights the magnitude attenuation and phase shift caused by frequency dependence of the observer proposed in [5]. It should be noted that $\omega_{0}$ in (7) is the same in the two cases. In case of non-linear loads, for instance the diode rectifier of Fig. 11, the designed observer does not show superior performance to the one that uses $\alpha \beta$-frame implementation. Fig. 17 shows the comparison between the two observers when a three-phase diode rectifier,
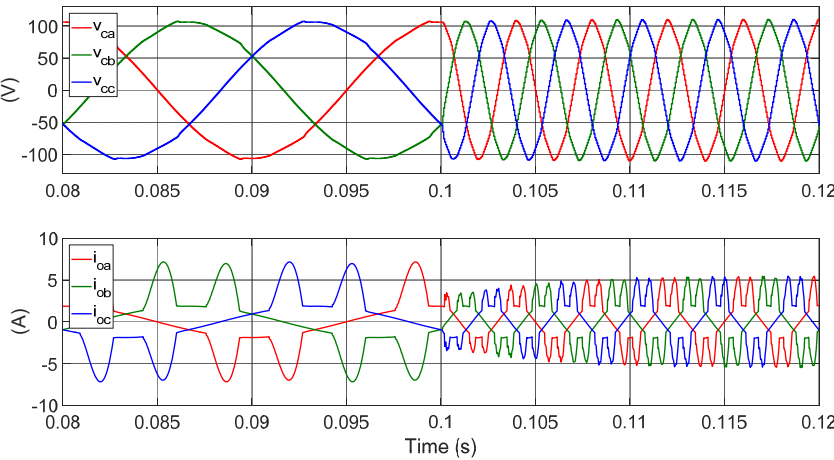

Fig. 15. Frequency step response with a combination of linear and non-linear loads - top: output phase voltages, bottom: load phase currents.
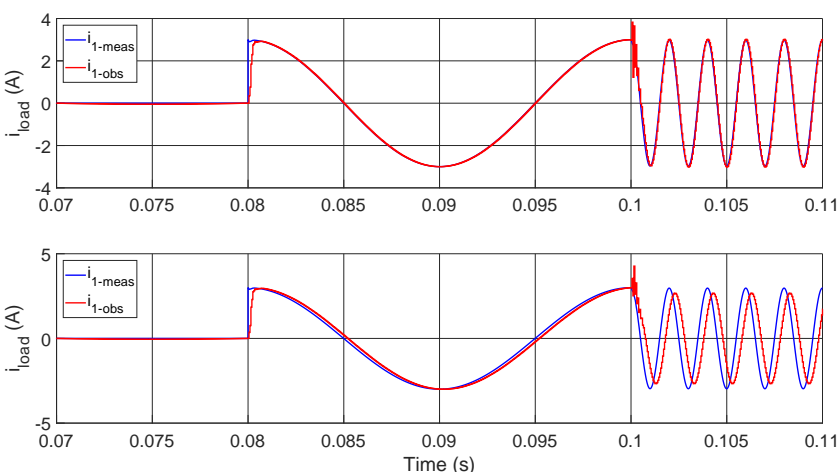

Fig. 16. Observed and measured load phase current comparison with magnitude and frequency step condition (resistive load) - top: with proposed observer, bottom: with the observer of [5].
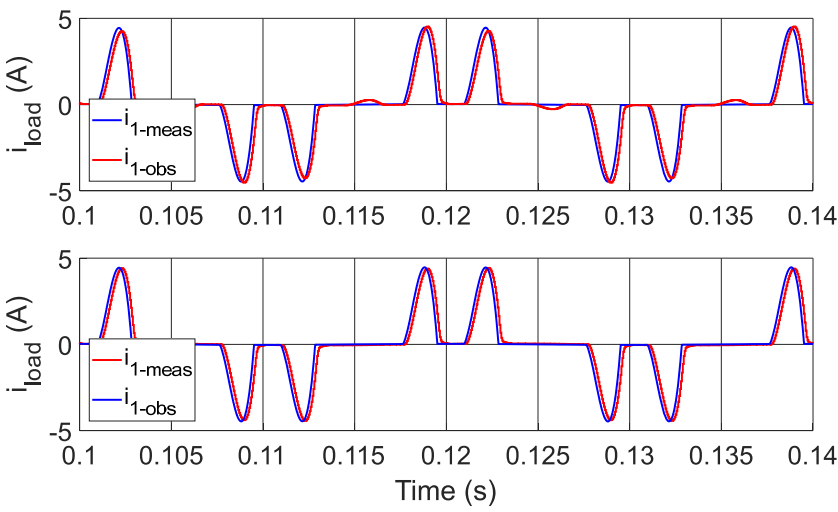

Fig. 17. Observed and measured load phase current comparison with nonlinear resistive load of Fig. 11 - top: with proposed observer, bottom: with the observer of [5].

with parallel $R C$ load on its dc-side, is used. The reason for the observer designed here not being as good as in case of linear loads is that the rectifier currents in $d q$ reference frame are not constant, rather they vary at six times the fundamental frequency (as shown in Fig 18) and the observer with gains of (7) does not have sufficient bandwidth to follow these fast varying currents. Nevertheless, the performance is comparable to the observer presented in the literature (Fig. 17 lower plot). 


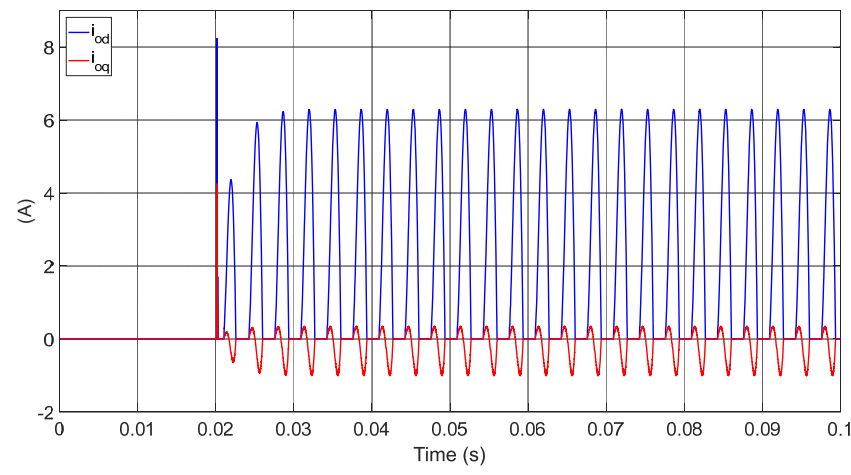

Fig. 18. Load currents in $d q$ reference frame for the non-linear load of Fig. 11.

\section{Conclusion}

The paper presented a modulated model predictive control for output voltage regulation of a voltage source inverter with LC output filter. The adopted control optimizes the duty cycles in linear regulation range and preserves the fast dynamics of a finite control set MPC in over-modulation. The control performance was enhanced by a load current observer that was implemented in $d q$ rotating reference frame instead of $\alpha \beta$-frame as usually done in the literature. The observer dynamics were shown to be independent of output frequency, which is an important result for programmable ac power supplies. Thanks to this feature of the designed observer, a step change in output reference frequency can be followed with the same dynamics as the load insertion step. The controller and observer performance was validated both in simulation and in experiments. Presented results showed good performance with linear and non-linear loads as well as a combination of them.

\section{REFERENCES}

[1] J. Rocabert, A. Luna, F. Blaabjerg, and P. Rodríguez, "Control of Power Converters in AC Microgrids," IEEE Transactions on Power Electronics, vol. 27, no. 11, pp. 4734-4749, nov 2012.

[2] E.-K. Kim, F. Mwasilu, H. H. Choi, and J.-W. Jung, "An Observer-Based Optimal Voltage Control Scheme for Three-Phase UPS Systems," IEEE Transactions on Industrial Electronics, vol. 62, no. 4, pp. 2073-2081, apr 2015.

[3] J. Steinke, "Use of an LC filter to achieve a motor-friendly performance of the PWM voltage source inverter," IEEE Transactions on Energy Conversion, vol. 14, no. 3, pp. 649-654, 1999.

[4] K. Hatua, A. K. Jain, D. Banerjee, and V. T. Ranganathan, "Active Damping of Output LC Filter Resonance for Vector-Controlled VSIFed AC Motor Drives," IEEE Transactions on Industrial Electronics, vol. 59, no. 1, pp. 334-342, jan 2012.

[5] P. Cortes, G. Ortiz, J. Yuz, J. Rodriguez, S. Vazquez, and L. Franquelo, "Model Predictive Control of an Inverter With Output LC Filter for UPS Applications," IEEE Transactions on Industrial Electronics, vol. 56, no. 6, pp. 1875-1883, jun 2009.

[6] H. T. Nguyen, E.-K. Kim, I.-P. Kim, H. H. Choi, and J.-W. Jung, "Model Predictive Control with Modulated Optimal Vector for a Three-Phase Inverter with an LC filter," IEEE Transactions on Power Electronics, vol. 33, no. 3, pp. 2690-2703, 2018.

[7] S. Vazquez, J. Leon, L. Franquelo, J. Carrasco, E. Dominguez, P. Cortes, and J. Rodriguez, "Comparison between FS-MPC control strategy for an UPS inverter application in $\alpha-\beta$ and abc frames," in 2010 IEEE International Symposium on Industrial Electronics. IEEE, jul 2010, pp. 3133-3138.
[8] F. de Bosio, L. A. Ribeiro, F. D. Freijedo, M. Pastorelli, and J. M. Guerrero, "Effect of state feedback coupling and system delays on the transient performance of stand-alone VSI with LC output filter," IEEE Transactions on Industrial Electronics, vol. 63, no. 8, pp. 4909 - 4918, 2016.

[9] S. Yang, Q. Lei, F. Z. Peng, and Z. Qian, "A Robust Control Scheme for Grid-Connected Voltage-Source Inverters," IEEE Transactions on Industrial Electronics, vol. 58, no. 1, pp. 202-212, jan 2011.

[10] V. Yaramasu, M. Rivera, M. Narimani, Bin Wu, and J. Rodriguez, "Model Predictive Approach for a Simple and Effective Load Voltage Control of Four-Leg Inverter With an Output LC Filter," IEEE Transactions on Industrial Electronics, vol. 61, no. 10, pp. 5259-5270, oct 2014.

[11] A. Sapin, P. K. Steimer, and J.-J. Simond, "Modeling, Simulation, and Test of a Three-Level Voltage-Source Inverter With Output LC Filter and Direct Torque Control," IEEE Transactions on Industry Applications, vol. 43, no. 2, pp. 469-475, 2007.

[12] J. He and Y. W. Li, "Generalized Closed-Loop Control Schemes with Embedded Virtual Impedances for Voltage Source Converters with LC or LCL Filters," IEEE Transactions on Power Electronics, vol. 27, no. 4, pp. 1850-1861, apr 2012.

[13] P. Loh and D. Holmes, "Analysis of Multiloop Control Strategies for LC/CL/LCL-Filtered Voltage-Source and Current-Source Inverters," IEEE Transactions on Industry Applications, vol. 41, no. 2, pp. 644-654, mar 2005.

[14] H. A. Young, M. A. Perez, J. Rodriguez, and H. Abu-Rub, "Assessing Finite-Control-Set Model Predictive Control: A Comparison with a Linear Current Controller in Two-Level Voltage Source Inverters," IEEE Industrial Electronics Magazine, vol. 8, no. 1, pp. 44-52, mar 2014.

[15] P. Karamanakos, T. Geyer, N. Oikonomou, F. D. Kieferndorf, and S. Manias, "Direct Model Predictive Control: A Review of Strategies That Achieve Long Prediction Intervals for Power Electronics," IEEE Industrial Electronics Magazine, vol. 8, no. 1, pp. 32-43, mar 2014.

[16] S. Kouro, M. A. Perez, J. Rodriguez, A. M. Llor, and H. A. Young, "Model Predictive Control: MPC's Role in the Evolution of Power Electronics," IEEE Industrial Electronics Magazine, vol. 9, no. 4, pp. 8 $-21,2015$.

[17] R. P. Aguilera and D. E. Quevedo, "Predictive control of power converters: Designs with guaranteed performance," IEEE Transactions on Industrial Informatics, vol. 11, no. 1, pp. 53 - 63, 2015.

[18] S. Vazquez, J. I. Leon, L. G. Franquelo, J. Rodriguez, H. A. Young, A. Marquez, and P. Zanchetta, "Model Predictive Control: A Review of Its Applications in Power Electronics," IEEE Industrial Electronics Magazine, vol. 8, no. 1, pp. 16-31, mar 2014.

[19] L. Tarisciotti, P. Zanchetta, A. Watson, J. C. Clare, M. Degano, and S. Bifaretti, "Modulated Model Predictive Control for a Three-Phase Active Rectifier," IEEE Transactions on Industry Applications, vol. 51, no. 2, pp. 1610-1620, 2015.

[20] S. Odhano, R. Bojoi, A. Formentini, P. Zanchetta, and A. Tenconi, "Direct flux and current vector control for induction motor drives using model predictive control theory," IET Electric Power Applications, vol. 11, no. 8, pp. 1483-1491, 2017.

[21] S. Vazquez, A. Marquez, J. I. Leon, L. G. Franquelo, and T. Geyer, "FCS-MPC and observer design for a VSI with output LC filter and sinusoidal output currents," in 2017 11th IEEE International Conference on Compatibility, Power Electronics and Power Engineering (CPEPOWERENG). IEEE, 2017, pp. 677-682.

[22] E. Fuentes, C. A. Silva, and R. M. Kennel, "MPC Implementation of a Quasi-Time-Optimal Speed Control for a PMSM Drive, With Inner Modulated-FS-MPC Torque Control," IEEE Transactions on Industrial Electronics, vol. 63, no. 6, pp. 3897-3905, jun 2016.

[23] C. F. Garcia, C. A. Silva, J. R. Rodriguez, P. Zanchetta, and S. A. Odhano, "Modulated Model Predictive Control with Optimized Overmodulation," IEEE Journal of Emerging and Selected Topics in Power Electronics, pp. 1-1, 2018.

[24] Chroma ATE Inc., "AC Power Source MODEL 61600 SERIES,” pp. 1 $-4,2017$.

[25] P. Cortes, J. Rodriguez, C. Silva, and A. Flores, "Delay Compensation in Model Predictive Current Control of a Three-Phase Inverter," IEEE Transactions on Industrial Electronics, vol. 59, no. 2, pp. 1323-1325, feb 2012.

[26] O. Troeng, B. Bernhardsson, and C. Rivetta, "Complex-coefficient systems in control," in 2017 American Control Conference (ACC). IEEE, may 2017, pp. 1721-1727. 